\title{
SGLT-2 inhibitors and cardiorenal outcomes in patients with or without type 2 diabetes: a meta-analysis of 11 CVOTs
}

Dario Giugliano ${ }^{1,2^{*}} \mathbb{D}$, Miriam Longo ${ }^{1}$, Lorenzo Scappaticcio², Giuseppe Bellastella ${ }^{1,2}$, Maria Ida Maiorino ${ }^{2,3}$ and Katherine Esposito 2,3

\begin{abstract}
Background: It has been suggested that sodium-glucose cotransporter 2 (SGLT-2) inhibitors reduce the cardiorenal risk in patients with type 2 diabetes (T2D). The purpose of this study is to provide an update of all large cardiovascular outcome trials (CVOTs) with SGLT-2 inhibitors to assess their cardiorenal efficacy in patients with and without T2D.

Methods: An electronic search up to 30 September 2021 was conducted in PubMed, EMBASE, the Cochrane Database of Systematic Reviews, and ClinicalTrials.gov. to determine eligible trials. We included CVOTs comparing any SGLT-2 inhibitor with placebo, reporting desired cardiovascular or renal outcomes and with a follow-up duration of at least 6 months.

Results: Eleven CVOTs, with data from five SGLT-2 inhibitors (empagliflozin, canagliflozin, dapagliflozin, ertugliflozin and sotagliflozin) and 77,541 participants, were included. In the overall analysis, the risk of the composite CV mortality or hospitalization for heart failure (HF) was reduced by $23 \%(\mathrm{HR}=0.77,95 \% \mathrm{Cl} 0.73-0.82, \mathrm{P}<0.001)$ compared with placebo, with not significant heterogeneity $\left(I^{2}=26 \%, P=0.20\right)$, and irrespective of the presence of T2D ( $P$ for interaction $=0.81$ ) and age ( $>65 \mathrm{vs} \leq 65$ years, $P$ for interaction $=0.78)$. The risk of $C V$ mortality, total mortality and hospitalization for HF was significantly reduced by $16 \%, 13 \%$, and $32 \%$, respectively; similarly, the risk of the composite renal outcome was reduced by $35 \%(\mathrm{HR}=0.65,95 \% \mathrm{Cl} 0.56-0.75)$, with moderate heterogeneity $\left(\mathrm{I}^{2}=32 \%\right)$. In the analysis of 6 CVOTs reporting the data, the risk of major cardiovascular events (MACE) was reduced by $12 \%$, with low heterogeneity $\left(I^{2}=21.2 \%, P=0.19\right)$ and irrespective of the presence of established $C V$ disease at baseline ( $P$ for interaction $=0.46)$.
\end{abstract}

Conclusions: Therapy with SGLT-2 inhibitors in patients with cardiometabolic and renal diseases results in a sustained to moderate reduction of the composite $\mathrm{CV}$ death or hospitalization for $\mathrm{HF}$, robust reduction of $\mathrm{HF}$ and renal outcomes, moderate reduction of CV mortality, total mortality and MACE.

Keywords: Cardiovascular outcome trials, Type 2 diabetes, SGLT-2 inhibitors, Cardiorenal outcomes

*Correspondence: dario.giugliano@unicampania.it

${ }^{1}$ Division of Endocrinology and Metabolic Diseases, Department

of Advanced Medical and Surgical Sciences, University of Campania "Luigi Vanvitelli", Naples, Italy

Full list of author information is available at the end of the article

\section{Introduction}

Inhibitors of the sodium-glucose co-transporter 2 (SGLT-2) were initially developed for the treatment of type 2 diabetes (T2D) for their effect of lowering blood glucose levels through increased excretion of glucose in the urine [1]. In FDA-mandated cardiovascular safety trials, also identified as cardiovascular outcome (CVOTs) original author(s) and the source, provide a link to the Creative Commons licence, and indicate if changes were made. The images or other third party material in this article are included in the article's Creative Commons licence, unless indicated otherwise in a credit line to the material. If material is not included in the article's Creative Commons licence and your intended use is not permitted by statutory regulation or exceeds the permitted use, you will need to obtain permission directly from the copyright holder. To view a copy of this licence, visit http://creativecommons.org/licenses/by/4.0/. The Creative Commons Public Domain Dedication waiver (http://creativeco mmons.org/publicdomain/zero/1.0/) applies to the data made available in this article, unless otherwise stated in a credit line to the data. 
trials [2], patients with T2D were typically divided by the presence or absence of established cardiovascular disease. However, once their efficacy in reducing the risk of heart failure or progression of chronic kidney disease was ascertained [3-6] and thought to be largely independent of baseline and time-dependent changes in glycated hemoglobin [7], the hypothesis emerged that their cardiorenal benefits might not necessarily be due to glucose lowering and might also be found in individuals without diabetes. The DAPA-HF (Dapagliflozin And Prevention of Adverse Outcomes in Heart Failure) trial [8] was the first one to include patients with heart failure and reduced ejection fraction irrespective of the presence of T2D: the trial showed that dapagliflozin reduced the risk of worsening heart failure events and cardiovascular death and improved symptoms in patients with or without T2D.

Since then, other CVOTs have been published which have extended the number and the typology of patients candidate to this class of drugs, giving more information to clinicians to optimize therapy and hopefully reduce their risk of cardiorenal complications. One key question is how the effects of SGLT-2 inhibitors compared in patients with and without T2D, and whether the findings of the completed trials support the hypothesis that SGLT-2 inhibition might be an effective treatment for patients with heart failure, including those without diabetes. The aim of this meta-analysis was to provide an update of all large CVOTs with SGLT-2 inhibitors to assess their cardiorenal efficacy in patients with and without T2D.

\section{Methods}

\section{Search strategy and study selection}

This systematic review was based on PRISMA (Preferred Reporting Items for Systematic Reviews and Meta-Analyses) guidelines [9]. The protocol has not been registered in any platform. We searched PubMed, EMBASE, the Cochrane Database of Systematic Reviews, and ClinicalTrials.gov (http://www.clinicaltrials.gov) to identify all eligible trials comparing the efficacy of SGLT-2 inhibitors with that of placebo in adult patients with or without T2D. The terms used for the research were "sodium-glucose co-transporter 2 inhibitors", "empagliflozin", "canagliflozin", “dapagliflozin”, “ertugliflozin”, "sotagliflozin”, "placebo", "cardiorenal outcomes", "kidney outcomes", "MACE", "heart failure", and "randomized controlled trials". The search was filtered to include only randomized controlled trials (RCTs) or meta-analyses of human data. Searches were done up until September 30, 2021. We excluded observational non-randomized studies, registries, ongoing trials without results, duplicate series, meta-analysis, abstracts, and oral communications. Data were extracted by D.G. and M.L., with conflicts over study inclusion resolved by consensus. The prespecified selection criteria included: (1) randomized controlled trials comparing any SGLT-2 inhibitor with placebo; (2) RCTs reporting desired cardiovascular or renal outcomes; (3) RCTs completed before the FDA guidance of 2008 [2] and (4) follow-up duration of at least 6 months.

\section{Data extraction and quality assessment}

Results in trial reports (primary trial results and subsequent secondary publications), and their accompanying supplementary materials, were used as the primary source of information. The retrieved data included study characteristics, characteristics of patients, interventions, and outcome measures, that included the hazard ratios (HR) and confidence intervals (CI) for cardiorenal outcomes. In more recent trials examining the effects of some SGLT-2 inhibitors (dapagliflozin or empagliflozin) independent of the presence of T2D, we did subgroups analysis assessing the effect of the SGLT-2 inhibitors on the primary outcome (CV death or hospitalization for $\mathrm{HF}$ ) in subjects with T2D vs subjects without T2D, or in subjects $>65$ years vs. subjects $\leq 65$ years of age. The Cochrane Collaboration Risk-of-Bias tool was used for quality assessment of the RCTs [10], including sequence generation, allocation concealment, blinding, incomplete outcome data, and selective outcome reporting. Risk of bias was graded as unclear, high, or low.

\section{Data synthesis and analysis}

Data were analyzed using Stata, version 16.0 (Stata Corp., College Station, TX). All statistical tests were two-sided, and $P$ values $<0.05$ were regarded as significant. The efficacy outcomes for this meta-analysis were the effect of SGLT-2 inhibitors on the incidence of the composite of $\mathrm{CV}$ mortality or hospitalization for HF, CV mortality, total mortality, hospitalization for HF, kidney outcomes and MACE. Subgroup analyses were done for the incidence of the composite of CV mortality or hospitalization for HF according to the presence of T2D at baseline (YES vs NO) or in subjects of 65 years of age or younger vs patients older than 65 years of age, as well as for incidence of MACE according to the presence of CV disease at baseline (YES vs NO). Hazard ratios (HRs) and 95\% CI (confidence interval) for efficacy outcomes were synthesized. Heterogeneity between studies was evaluated by using the Cochran's $Q$ test. The proportion of variation in observed effects due to heterogeneity rather than sampling error was evaluated by using $\mathrm{I}^{2}$ index [11] and thresholds of $\mathrm{I}^{2}$ describing the degree of heterogeneity were $25 \%$ or lower (low), $26-75 \%$ (moderate), and greater than $75 \%$ (high). A Q statistic P-value of $<0.10$ was considered significant Pooled summary estimates and $95 \%$ 
CIs for efficacy outcomes were calculated according to a random effects model using the Paule-Mandel method [12]. Publication bias was assessed with the Egger test [13]. The trim-and-fill method [14] was used to estimate the effect of publication bias (if any).

\section{Results}

\section{Characteristics of the included studies}

Of 160 articles screened for eligibility, 11 RCTs [8, 15-29] were eligible and included in the meta-analysis (Additional file 1: Fig. S1). Their characteristics are summarized in Table 1 . The participants were all adult ( $>18$ years old) patients. All trials were multinational and sponsored by industry. The trials have been published between 2015 and 2021, with 3 trials published in 2021 . All trials were of parallel-group, double-blind design, and their mean duration ranged from 0.75 to 4.2 years. The populations studied ranged in size from 1222 (SOLOISTWHF) to 17,160 (DECLARE) and were of similar age (range 61.3-71.9 years). Data from 77,541 participants were included in the analysis.

EMPA-REG OUTCOME compared empagliflozin to placebo in 7020 patients with T2D and established CV disease [15, 25]. CANVAS compared canagliflozin to placebo in 10,142 patients with T2D and established CV disease or CV risk factors only [16]. DECLARE compared dapagliflozin to placebo in 17,160 patients with T2DM and established CV disease or CV risk factors only [17]. CREDENCE compared canagliflozin to placebo in 4401 patients with T2D and diabetic kidney disease $[18,26]$. DAPA-HF compared dapagliflozin to placebo in 4744 patients with or without T2D and heart failure with reduced ejection fraction $[8,27$, 28]. DAPA-CKD compared dapagliflozin to placebo in 4304 patients with or without T2D and chronic kidney disease [19, 29]. VERTIS-CV compared ertugliflozin to placebo in 8246 patients with T2D and established $\mathrm{CV}$ disease [20]. EMPEROR-R compared empagliflozin to placebo in 3730 patients with or without T2D and heart failure with reduced ejection fraction [21]. SCORED compared sotagliflozin to placebo in 10,584 patients with T2D and chronic kidney disease [22]. SOLOIST-WHF compared sotagliflozin to placebo in 1222 patients with T2D who were recently hospitalized for worsening heart failure [23]. EMPEROR-P compared empagliflozin to placebo in 5988 patients with or without T2D and heart failure with preserved ejection fraction [24]. The primary outcomes for the 11 trials are given in Table 1. According to the Cochrane Collaboration's tool for assessing risk of bias, there was no major risk of bias in any study (Additional file 1: Fig. S2, Table S1).

Table 1 Characteristics of CVOTs included in the meta-analysis

\begin{tabular}{|c|c|c|c|c|c|}
\hline & $\begin{array}{l}\text { Study drug/mean } \\
\text { follow-up (years) }\end{array}$ & Participants (n) & $\begin{array}{l}\text { Age, mean } \\
\text { or median } \\
\text { (years) }\end{array}$ & Male sex $(n, \%)$ & Primary outcome \\
\hline $\begin{array}{l}\text { EMPA-REG } \\
2015\end{array}$ & $\begin{array}{l}\text { Empagliflozin } \\
3.1\end{array}$ & 7020 & 61.3 & $\begin{array}{l}5016 \\
71.5\end{array}$ & MACE \\
\hline $\begin{array}{l}\text { CANVAS } \\
2017\end{array}$ & $\begin{array}{l}\text { Canagliflozin } \\
2.4\end{array}$ & 10,142 & 63.3 & $\begin{array}{l}6509 \\
64.2 \%\end{array}$ & MACE \\
\hline $\begin{array}{l}\text { DECLARE } \\
2019\end{array}$ & $\begin{array}{l}\text { Dapagliflozin } \\
4.2\end{array}$ & 17,160 & 63.9 & $\begin{array}{l}10,738 \\
62.6 \%\end{array}$ & MACE \\
\hline $\begin{array}{l}\text { CREDENCE } \\
2019\end{array}$ & $\begin{array}{l}\text { Canagliflozin } \\
2.6\end{array}$ & 4401 & 63.0 & $\begin{array}{l}2907 \\
66.1 \%\end{array}$ & $\begin{array}{l}\text { Composite renal: ESKD, doubling of serum creatinine levels, } \\
\text { death from renal or CV causes }\end{array}$ \\
\hline $\begin{array}{l}\text { DAPA-HF } \\
2019\end{array}$ & $\begin{array}{l}\text { Dapagliflozin } \\
1.5\end{array}$ & 4744 & 66.0 & $\begin{array}{l}3131 \\
66.0 \%\end{array}$ & Composite of worsening HF or CV death \\
\hline $\begin{array}{l}\text { DAPA CKD } \\
2020\end{array}$ & $\begin{array}{l}\text { Dapagliflozin } \\
2.4\end{array}$ & 4304 & 61.8 & $\begin{array}{l}2879 \\
66.9 \%\end{array}$ & $\begin{array}{l}\text { Composite renal: decline eGFR } \geq 50 \% \text {, ESKD, death from CV } \\
\text { or renal causes }\end{array}$ \\
\hline $\begin{array}{l}\text { VERTIS-CV } \\
2020\end{array}$ & $\begin{array}{l}\text { Ertugliflozin } \\
3.0\end{array}$ & 8246 & 64.4 & $\begin{array}{l}5769 \\
70.0 \%\end{array}$ & MACE \\
\hline $\begin{array}{l}\text { EMPEROR-R } \\
2020\end{array}$ & $\begin{array}{l}\text { Empagliflozin } \\
1.5\end{array}$ & 3730 & 66.8 & $\begin{array}{l}2837 \\
76.0 \%\end{array}$ & Composite of CV death and hospitalization for HF \\
\hline $\begin{array}{l}\text { SCORED } \\
2021\end{array}$ & $\begin{array}{l}\text { Sotagliflozin } \\
1.5\end{array}$ & 10,584 & 69.0 & $\begin{array}{l}5896 \\
55.7 \%\end{array}$ & $\begin{array}{l}\text { Composite of CV death, hospitalization for HF, urgent HT } \\
\text { for HF }\end{array}$ \\
\hline $\begin{array}{l}\text { SOLOIST-WHF } \\
2021\end{array}$ & $\begin{array}{l}\text { Sotagliflozin } \\
0.75\end{array}$ & 1222 & 70.0 & $\begin{array}{l}810 \\
66.3 \%\end{array}$ & $\begin{array}{l}\text { Composite of CV death, hospitalization for HF, urgent HT } \\
\text { for HF }\end{array}$ \\
\hline $\begin{array}{l}\text { EMPEROR-P } \\
2021\end{array}$ & $\begin{array}{l}\text { Empagliflozin } \\
2.2\end{array}$ & 5988 & 71.9 & $\begin{array}{l}3317 \\
55.4 \%\end{array}$ & Composite of CV death and hospitalization for HF \\
\hline
\end{tabular}

MACE: major adverse cardiovascular events; ESKD: end-stage kidney disease; CV: cardiovascular; HF: heart failure; eGRF: estimated glomerular filtration rate; HT: hospitalization 


\section{Outcomes}

In the overall analysis, including 11 trials with 77,541 participants, the risk of composite CV mortality or hospitalization for $\mathrm{HF}$ was reduced by $23 \%$ ( $\mathrm{HR}=0.77,95 \%$ CI $0.73-0.82, \mathrm{P}<0.001)$ compared with placebo, with moderate and not significant heterogeneity $\left(\mathrm{I}^{2}=26 \%\right.$, $\mathrm{P}=0.20$ ) (Fig. 1 and Table 2), and no evidence of publication bias (Egger test, $\mathrm{P}=0.46$ ). In the subanalysis of participants divided according to the presence or absence of T2D (Fig. 3 and Table 2), there was no difference in the risk of the composite $\mathrm{CV}$ death or hospitalization for HF between the two groups ( $\mathrm{P}$ for interaction $=0.81)$. In the four trials that included participants with or without T2D (DAPA-HF, DAPA-CKD, EMPEROR-R and EMPEROR-P), treatment with dapagliflozin (DAPA) or empagliflozin (EMPEROR) was associated with $26 \%(\mathrm{HR}=0.74,95 \%$ CI $0.64-0.84)$ and $23 \%(\mathrm{HR}=0.77,95 \% \mathrm{CI} 0.64-0.91)$ lower risk of the composite CV death or hospitalization for HF in patients with or without T2D, respectively. Similarly, in three trials (DAPA-HF, EMPEROR-R and EMPEROR-P) treatment with dapagliflozin or empagliflozin was associated with $25 \%(\mathrm{HR}=0.75,95 \%$ CI $0.66-0.84)$ and $22 \%$ $(\mathrm{HR}=0.78,95 \%$ CI $0.68-0.88)$ lower risk of the composite $\mathrm{CV}$ death or hospitalization for $\mathrm{HF}$ in patients older than 65 years or 65-year-old or younger, respectively, with no significant interaction $(\mathrm{P}=0.78)$ (Fig. 4 and Table 2).

In the overall analysis including all the 11 CVOTs, the risk of $\mathrm{CV}$ mortality (Fig. 5 and Table 2), was reduced by $16 \%(\mathrm{HR}=0.84,95 \% \mathrm{CI} 0.73-0.95)$ by treatment with SGLT-2 inhibitors, with moderate and significant heterogeneity $\left(\mathrm{I}^{2}=0.42 \%, \mathrm{P}=0.07\right)$ and some evidence of publication bias (Egger test, $\mathrm{P}=0.047$ ). The trim-and-fill method indicated that this publication bias did not change the statistical significance of the estimate (HR 0.85, 95\% CI 0.74-0.96). Similarly, the risk of total mortality (Fig. 6 and Table 2), was reduced by $13 \%(\mathrm{HR}=0.87,95 \% \mathrm{CI} 0.74-0.98)$ by treatment with SGLT-2 inhibitors, with moderate heterogeneity $\left(\mathrm{I}^{2}=0.45 \%\right)$ and some evidence of publication bias (Egger test, $\mathrm{P}=0.042$ ). The trim-and-fill method

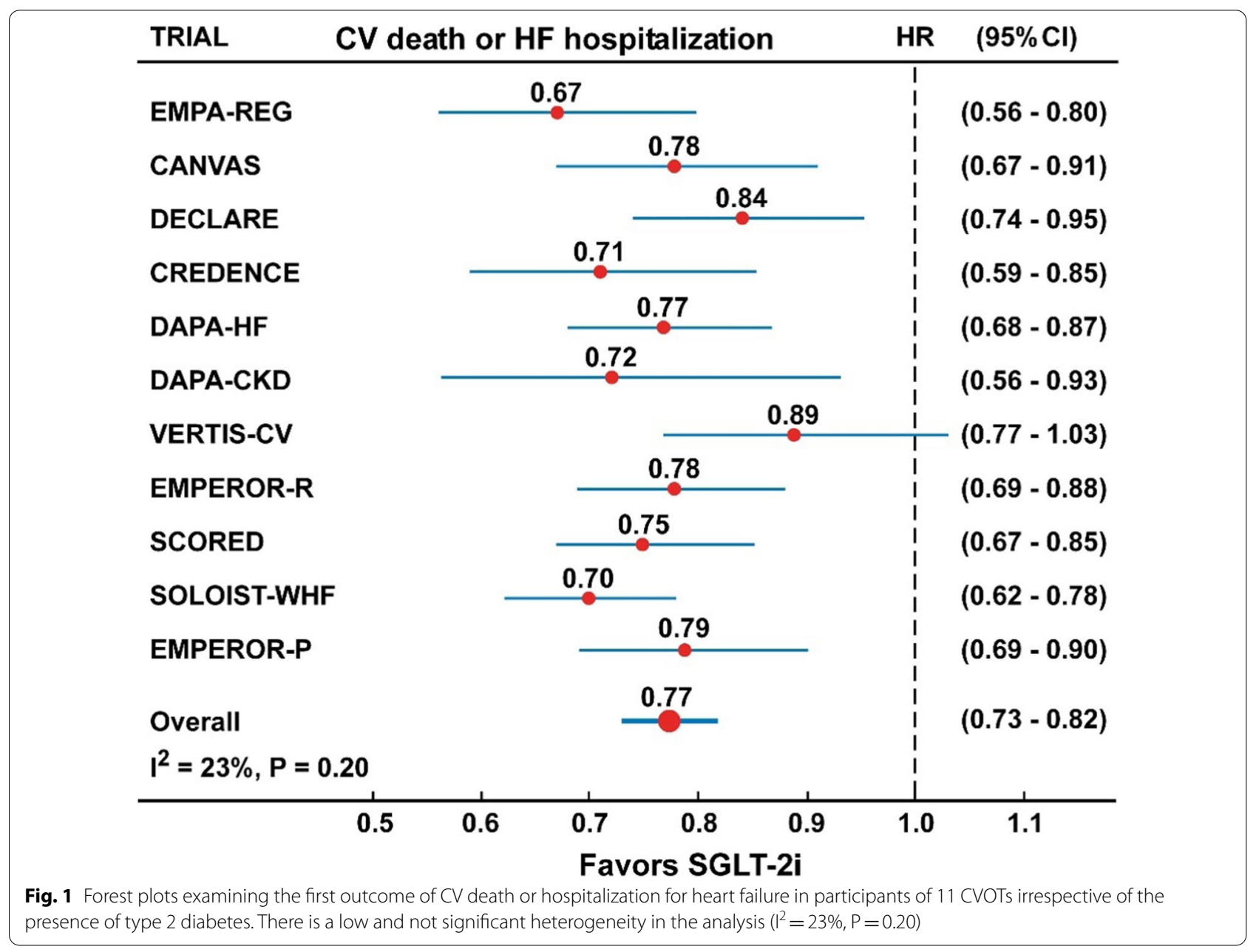


Table 2 Results of planned meta-analyses with random effects

\begin{tabular}{|c|c|c|c|c|c|c|}
\hline Outcome & Trials (n) & Estimate (HR) & $95 \% \mathrm{Cl}$ & $P$ value of $H R$ & $\mathrm{I}^{2}(\%)$ & P value of $I^{2}$ \\
\hline \multicolumn{7}{|l|}{$\mathrm{CV}$ death and $\mathrm{HFH}$} \\
\hline All & 11 & 0.77 & $0.73-0.82$ & $<0.001$ & 26.0 & 0.20 \\
\hline T2D: yes & 4 & 0.74 & $0.64-0.84$ & $<0.001$ & 0 & 0.81 \\
\hline T2D: no & 4 & 0.77 & $0.64-0.91$ & $<0.001$ & 0 & 0.72 \\
\hline Age $>65$ years & 3 & 0.75 & $0.66-0.84$ & $<0.001$ & 0 & 0.92 \\
\hline Age $\leq 65$ years & 3 & 0.78 & $0.68-0.88$ & $<0.001$ & 2.0 & 0.87 \\
\hline \multicolumn{7}{|l|}{ CV mortality } \\
\hline All & 11 & 0.84 & $0.73-0.95$ & 0.007 & 42.0 & 0.10 \\
\hline \multicolumn{7}{|l|}{ Total mortality } \\
\hline All & 11 & 0.87 & $0.74-0.98$ & 0.009 & 45.0 & 0.07 \\
\hline \multicolumn{7}{|l|}{ HF hospitalization } \\
\hline All & 10 & 0.68 & $0.62-0.74$ & $<0.001$ & 0 & 0.98 \\
\hline \multicolumn{7}{|l|}{ Kidney outcomes } \\
\hline All & 10 & 0.65 & $0.56-0.75$ & $<0.001$ & 35.0 & 0.10 \\
\hline \multicolumn{7}{|l|}{ MACE } \\
\hline All & 6 & 0.88 & $0.83-0.93$ & $<0.010$ & 21.2 & 0.19 \\
\hline Prior CVD & 5 & 0.87 & $0.82-0.92$ & 0.001 & 12.0 & 0.35 \\
\hline No prior CVD & 3 & 0.93 & $0.83-1.07$ & 0.326 & 55.1 & 0.10 \\
\hline
\end{tabular}

$\mathrm{CV}$, cardiovascular; $\mathrm{HFH}$, hospitalization for heart failure; $\mathrm{HR}$, hazard ratio; $\mathrm{Cl}$, confidence intervals; T2D, type 2 diabetes; MACE, major cardiovascular events

indicated that this publication bias did not change the statistical significance of the estimate (HR 0.88, 95\% CI 0.75-0.97).
In the analysis of 10 trials with SGLT-2 inhibitors (Fig. 7, Table 2), the risk of hospitalization for HF was reduced by $32 \%(\mathrm{HR}=0.68,95 \% \mathrm{CI} 0.62-0.74)$, with no

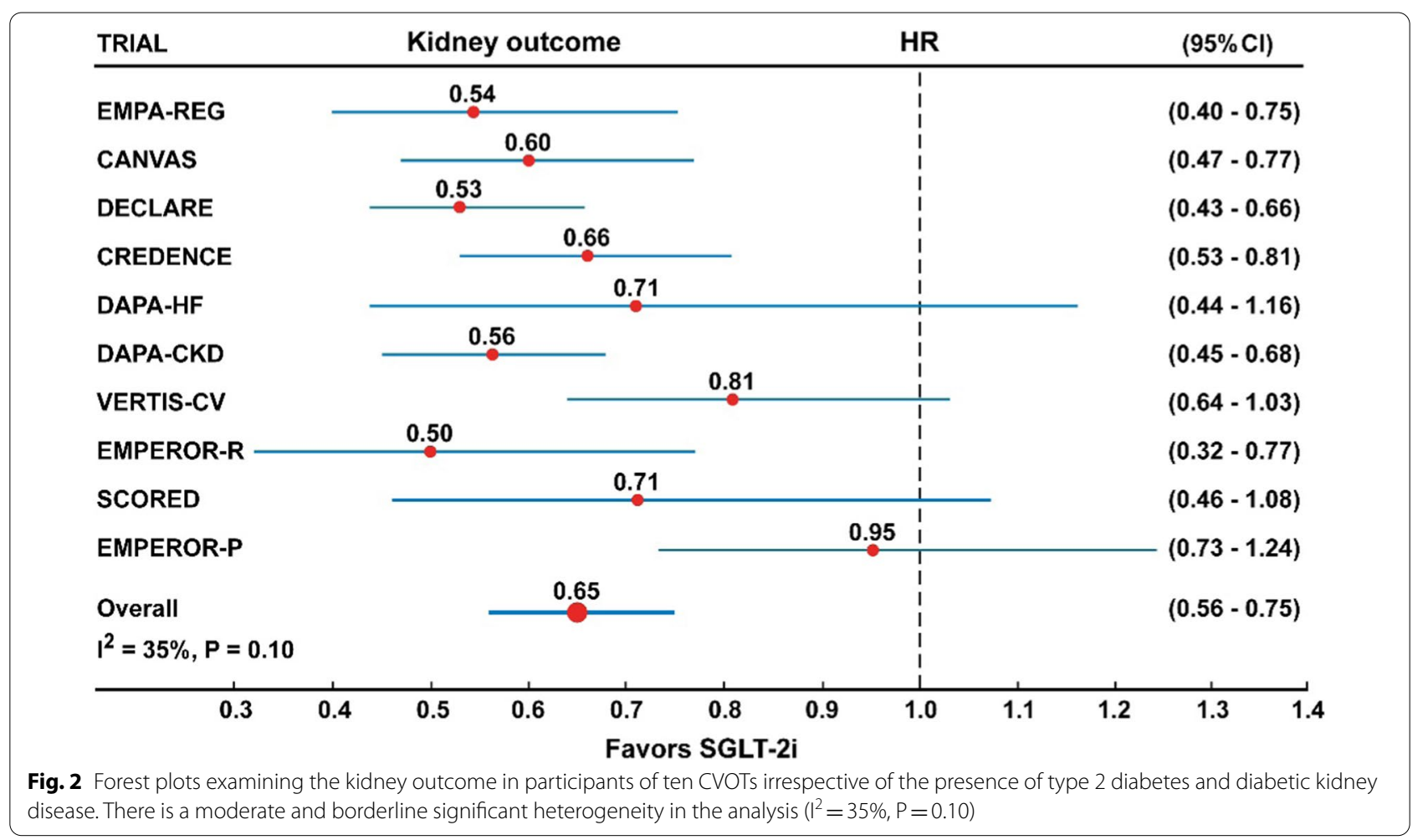




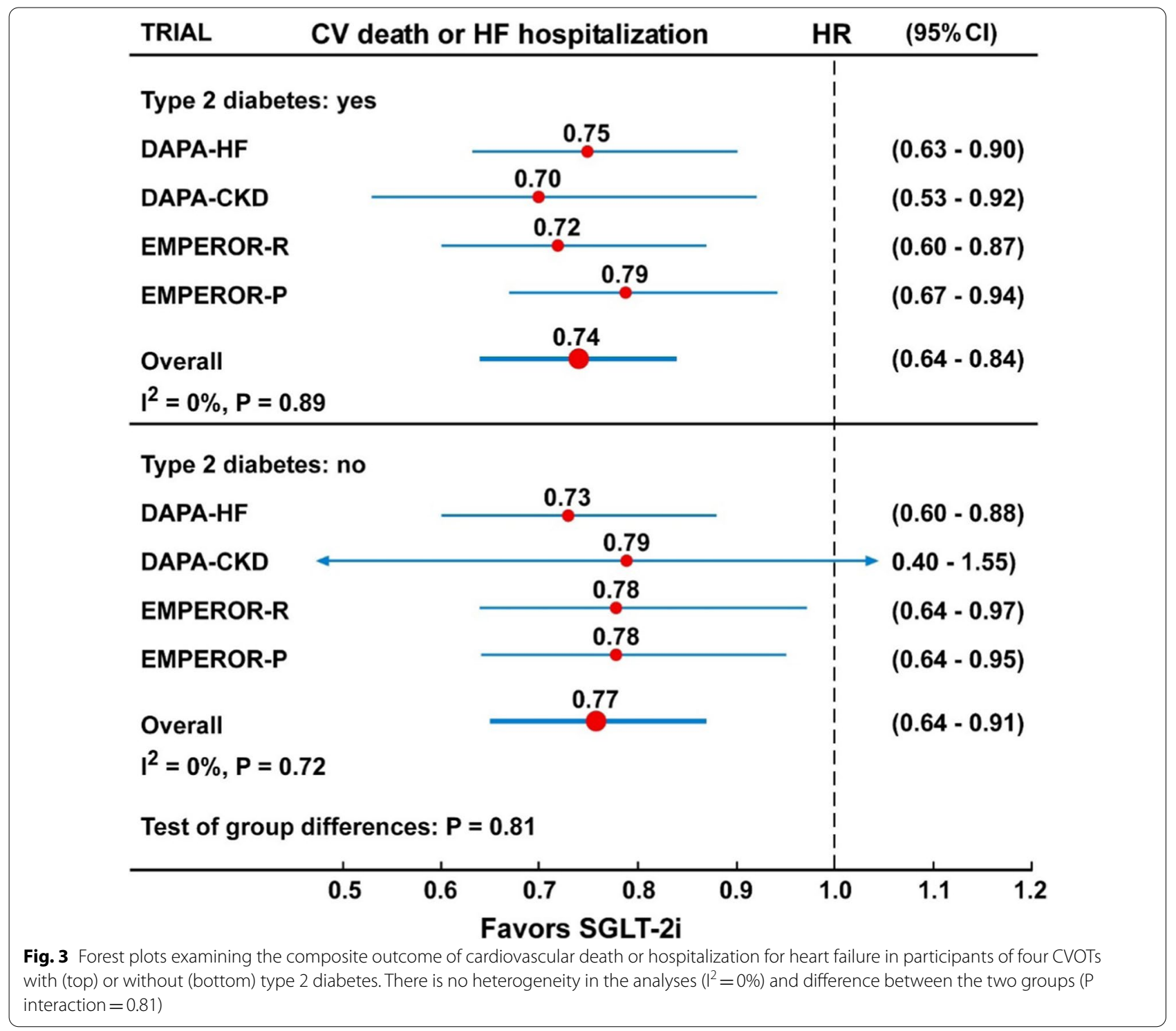

heterogeneity $\left(\mathrm{I}^{2}=0 \%\right)$ and no evidence of publication bias (Egger test, $\mathrm{P}=0.85$ ). Similarly, the risk of the composite renal outcome (Fig. 2, Table 2) was reduced by $35 \%$ $(\mathrm{HR}=0.65,95 \%$ CI $0.56-0.75)$, with moderate but not significant heterogeneity $\left(\mathrm{I}^{2}=35 \%\right)$ but no evidence of publication bias (Egger test, $\mathrm{P}=0.15$ ).

In the analysis of 6 CVOTs (EMPA-REG, CANVAS, DECLARE, CREDENE, VERTIS-CV, SCORED) (Table 2), the risk of MACE was reduced by $12 \%$, with low heterogeneity $\left(\mathrm{I}^{2}=21.2 \%, \mathrm{P}=0.19\right)$. There was no difference in the risk of MACE according to the presence or absence of established $\mathrm{CV}$ disease at baseline ( $\mathrm{P}$ for interaction $=0.46$ ).
In all analyses shown in Figs. 1, 2, 3, 4, 5, 6, 7, the Hazard ratios for the specific outcomes of two sotagliflozin trials (SCORED and SOLOIST-HF), when included, were like to those of the other SGLT-2 inhibitors (empagliflozin, canagliflozin, dapagliflozin, ertugliflozin).

\section{Discussion}

The present meta-analysis includes the most recent published large RCTs (SCORED, SOLOIST-WHF and EMPEROR-P), thus providing the most contemporary assessment of the total available evidence for SGLT-2 inhibitor therapy and cardiorenal outcomes in patients with or without T2D. The findings of 11 CVOTs involving 




77,541 patients show that treatment with SGLT-2 inhibitors reduced the risk of the composite $\mathrm{CV}$ death or hospitalization for $\mathrm{HF}$ by $23 \%$ in the overall population, with low and not significant heterogeneity, suggesting a plausible class effect for the outcome. This interpretation seems also supported by the subgroup analyses indicating the lack of significant difference in the reduced risk of the composite outcome in patients with or without T2D, or in subjects of 65 years of age or younger vs those older than 65 years of age. Although participants in the EMPEROR-P trial [24] were grouped by different age-threshold ( $>70$ years vs $\leq 70$ years), this may not have altered the estimation of the Hazard Ratio given the absence of heterogeneity and interaction between groups.

To date, the most meaningful class effect of SGLT-2 inhibitors appears to be that on HF hospitalization for the following reasons: (1) the reduced risk for HF hospitalization is $>25 \%$ in every CVOT published until now (range 27-39\%); (2) there is a complete absence of heterogeneity $\left(\mathrm{I}^{2}=0 \%, \mathrm{P}=0.98\right)$ in the meta-analysis; (3) the confidence intervals of the HR are very close to the point estimate; and (4) the reduced risk of hospitalization for HF is significant in every CVOTs. So, we can be confident that the beneficial effect of SGLT-2 inhibitors to reduce the risk of hospitalization for HF is a class effect and is independent of the diabetes status, heart status (presence or absence of HF or established CV disease at baseline) and kidney status (presence or absence of chronic kidney disease at baseline) [5, 30, 31].

A recent meta-analysis [32] of 8 CVOTs pooled data of 65,587 patients and found that treatment with SGLT-2 inhibitors reduced the risk of the renal composite endpoint by $39 \%$, including worsening renal function, 


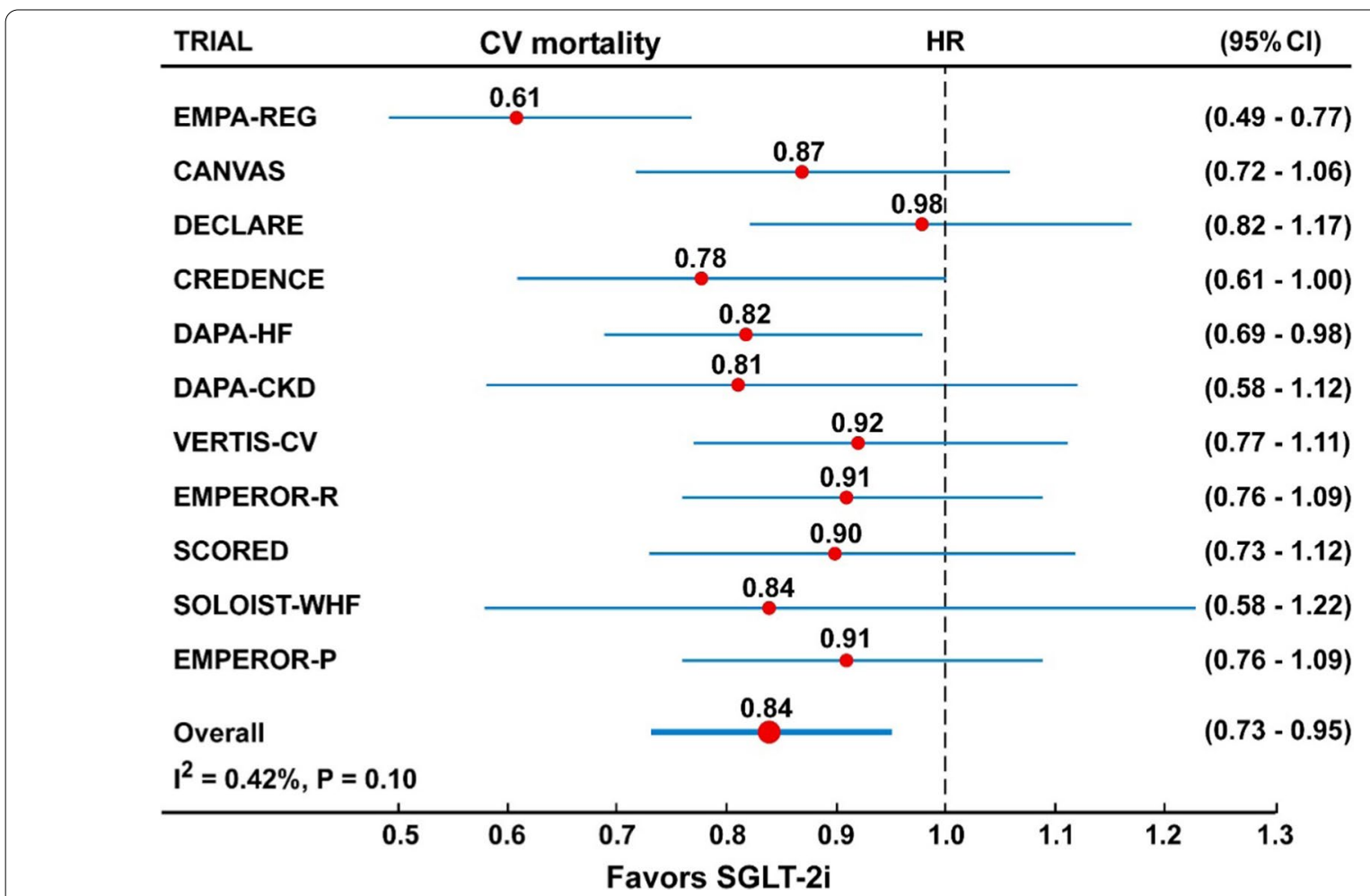

Fig. 5 Forest plots examining the outcome cardiovascular mortality in participants of 11 CVOTs. There is moderate heterogeneity in the analyses $\left(I^{2}=42 \%\right.$ of borderline significance $)$

end-stage kidney disease and death from $\mathrm{CV}$ or renal causes, adding evidence to their nephroprotective effect. While confirming nephroprotection by SGLT-2 inhibitors with a pooled reduced risk of renal outcome by $35 \%$, the present meta-analysis including ten CVOTs reporting the renal outcome seems to question the class effect of SGLT-2 inhibitors for the following reasons: (1) the reduced risk of renal outcome is not significant in every CVOTs; (2) apart from canagliflozin, all other inhibitors (dapagliflozin, ertugliflozin, sotagliflozin and empagliflozin) fail to produce a significant nephroprotection in at least one CVOT; (3) there is moderate and borderline significant heterogeneity $\left(\mathrm{I}^{2}=35 \%, \mathrm{P}=0.10\right)$ in the present meta-analysis, probably as a consequence of the different criteria used to define the kidney outcome; and (4) the worst results, in terms of wideness of confidence intervals, are observed in patients with HF, independent of the presence of T2D. In particular, the beneficial effect of empagliflozin on the renal outcome was significantly greater in the EMPEROR-R than in the EMPEROR$P$ trial [33], suggesting a major protective effect of the drug in patients with HF and a reduced ejection fraction. It is possible that the definition of the renal outcome may have played some role in the striking discordance between the effect of empagliflozin on heart failure and renal outcomes in EMPEROR-P trial. This discordance is extraordinarily puzzling as the effects of SGLT-2 inhibitors on hospitalization for HF and renal outcome had consistently tracked together in previous CVOTs. As empagliflozin reduced the risk of HF hospitalization in these two populations of patients with HF (reduced or preserved ejection fraction) irrespective of their baseline diabetic status, it is conceivable that nephroprotection is not the principal mechanism by which empagliflozin may prevent HF hospitalization [34]. It has already been shown that SGLT-2 inhibitors led to greater benefits in patients with NYHA class II than in patients with NYHA class III or IV, and that the reduced HF composite outcome is independent of LVEF level $(<40 \%, 40 \%$ to $<50 \%$, or $\geq 50 \%)[35,36]$.

Several hypotheses have been formulated in the attempt to explain the cardiorenal protective effects of SGLT-2 inhibitors, including, although not limited to a diuretic effect [37], altered substrate utilization and cellular signaling though increased lipolysis in adipose tissue with subsequent generation of ketone bodies [38], 


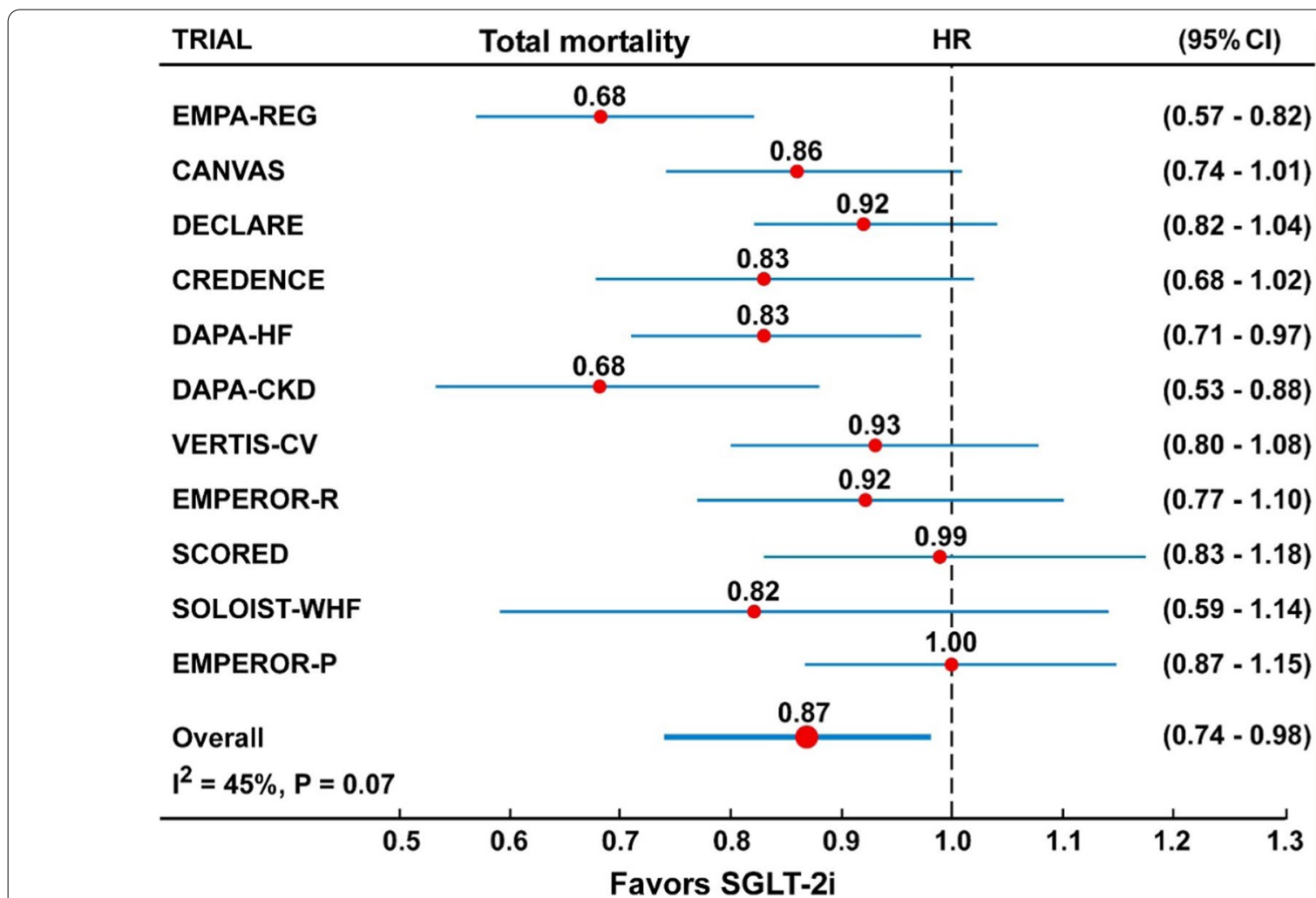

Fig. 6 Forest plots examining the outcome cardiovascular mortality in participants of 11 CVOTs. There is moderate and significant heterogeneity in the analyses $\left(1^{2}=45 \%\right)$

increased erythropoietin, hemoglobin, and hematocrit levels which can improve tissue oxygenation [39] and improved the lipid profile and decreasing uric acid level [40]. Moreover, the increase in the delivery of sodium to the macula densa results in vasoconstriction of the afferent arteriolar with a subsequent reduction in the intraglomerular pressure [41]. Irrespective of the exact mechanism, the improvement in cardiorenal outcomes by SGLT-2 inhibitors in patients with and without T2D suggest inherent protective properties. To date, it is not yet possible to clearly identify subpopulations of patients without T2D that would benefit the most from treatment with SGLT-2 inhibitors.

This study has potential limitations that include the use of aggregate trial-data levels and some difference in the exact inclusion/exclusion criteria and definition of outcomes among trials. Moreover, not all CVOTs have published the subgroup data for all outcomes and therefore some trials are not included in the analysis for individual endpoints. Strengths of the present meta-analysis are the inclusion of all CVOTs published by September 30,2021 , the very large number of participants, the high quality of the trials which minimizes the risk of bias, and the absence of significant heterogeneity in most analyses, which ranged from absent to low or moderate.

The clinical relevance of these results seems also highlighted by the evidence that for some outcomes the clinical benefit is consistent irrespective of the presence of $\mathrm{T} 2 \mathrm{D}$, advanced age, and cardiorenal disease. On the basis of results of CVOTs, the FDA has approved dapagliflozin (May 5, 2020) and empagliflozin (August 18, 2021) to reduce risk for $\mathrm{CV}$ death and $\mathrm{HF}$ hospitalization in adults with HF with reduced ejection fraction regardless of whether they have diabetes $[42,43]$.

\section{Conclusions}

Therapy with SGLT-2 inhibitors results in a sustained to moderate reduction of the composite CV death or hospitalization for $\mathrm{HF}$, robust reduction of $\mathrm{HF}$ and renal outcome, and moderate reduction of $\mathrm{CV}$, total mortality and MACE. The shift from a common antihyperglycemic drug to an agent with the likely indication of cardiorenal protection is under way and close to the goal. However, it is unlikely that future studies will significantly change the present scenario based on 11 CVOTs with more than 75,000 patients. 


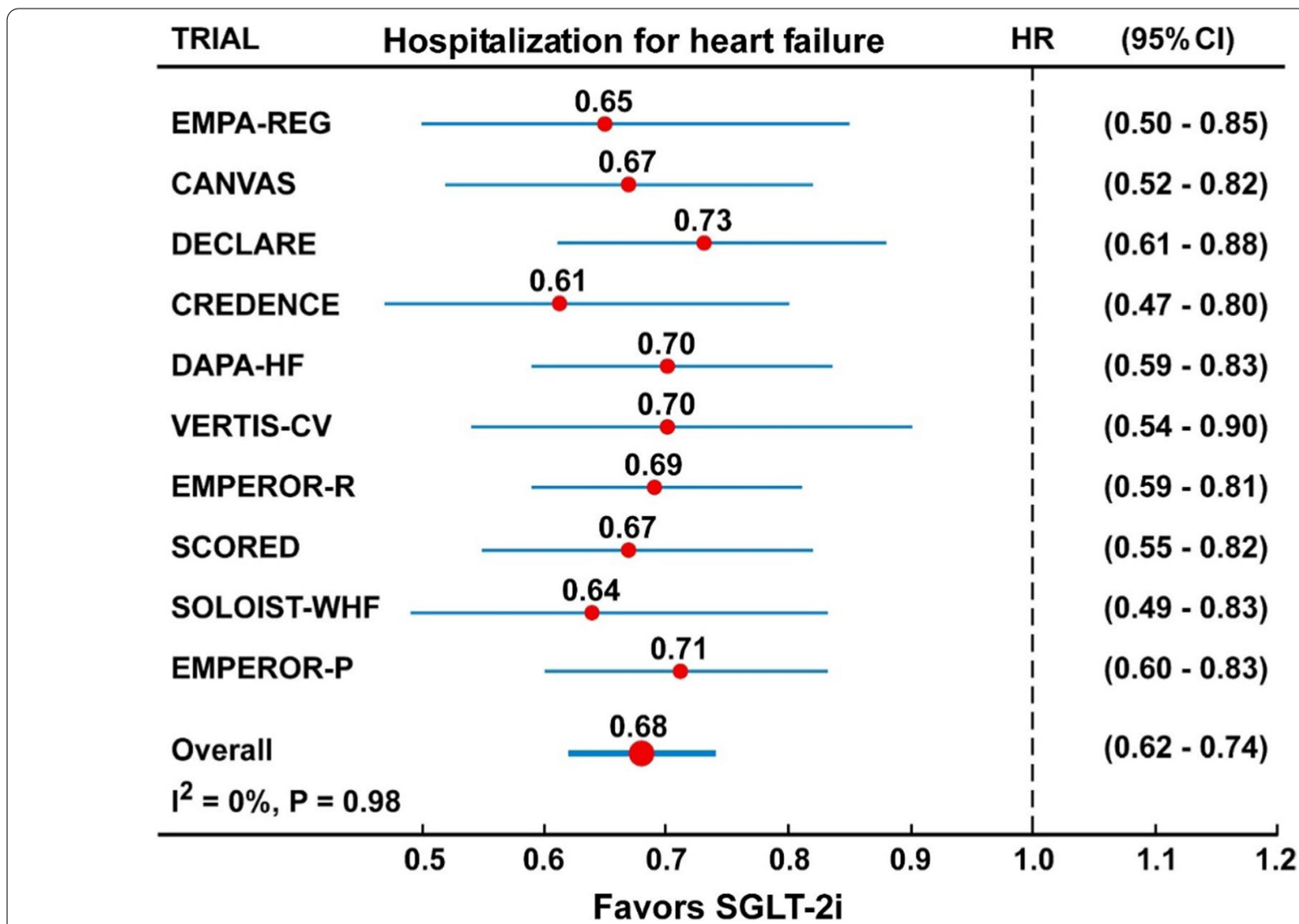

Fig. 7 Forest plots examining the outcome hospitalization for heart failure in participants of ten CVOTs. There is a null heterogeneity in the analyses $\left(1^{2}=0 \%\right)$

\section{Abbreviations}

CVOTs: Cardiovascular outcome trials; SGLT-2: Sodium-glucose cotransporter-2; T2D: Type 2 diabetes; MACE: Major cardiovascular events; HR: Hazard ratios; HF: Heart failure.

\section{Supplementary Information}

The online version contains supplementary material available at https://doi. org/10.1186/s12933-021-01430-3.

Additional file 1. Supplemental file.

Acknowledgements

Not applicable.

\section{Authors' contributions}

DG and KE designed the research. MIM, ML and LS collected data in electronic database. DG, ML, and MIM performed statistical analysis. All authors contributed to drafting of the manuscript, had full access to all the data in the study, approved the final version of the manuscript and had final decision to submit for publication. DG is the guarantor of this work and, as such, had full access to all the data in the study and takes responsibility for the integrity of the data and the accuracy of the data analysis. All authors read and approved the final manuscript.
Funding

None.

Availability of data and materials

All data generated or analyzed during this study are included in this published article and in its Additional file.

\section{Declarations}

Ethics approval and consent to participate Not applicable.

Consent for publication

Not applicable.

\section{Competing interests}

DG received honoraria for speaking at meetings from Novartis, Sanofi, Lilly, MundiPharma, Astrazeneca, and NovoNordisk. MIM received honoraria for speaking at meetings from Astrazeneca, NovoNordisk, Bruno, Mundipharma, Sanofi. KE received honoraria for speaking at meetings from Novartis, Sanofi-Aventis, Lilly, AstraZeneca, MundiPharma, Boehringer Ingelheim, and NovoNordisk. No other potential competing interest relevant to this article was reported.

\section{Author details}

'Division of Endocrinology and Metabolic Diseases, Department of Advanced Medical and Surgical Sciences, University of Campania "Luigi Vanvitelli", Naples, Italy. ${ }^{2} \mathrm{PhD}$ of Translational Medicine, Department of Advanced Medical and Surgical Sciences, University of Campania “Luigi Vanvitelli", Naples, Italy. 
${ }^{3}$ Diabetes Unit, Department of Advanced Medical and Surgical Sciences, University of Campania "Luigi Vanvitelli", Naples, Italy.

Received: 3 November 2021 Accepted: 7 December 2021 Published online: 16 December 2021

\section{References}

1. Pfister M, Whaley JM, Zhang L, List JF. Inhibition of SGLT2: a novel strategy for treatment of type 2 diabetes mellitus. Clin Pharmacol Ther. 2011;89:621-5.

2. Food and Drug Administration. Guidance for industry: diabetes mellitus - evaluating cardiovascular risk in new antidiabetic therapies to treat type 2 diabetes. Silver Spring: Food and Drug Administration; 2008. http://www.fda.gov/downloads/Drugs/Guidances/ucm071627.pdf. Accessed 24 Aug 2021.

3. Giugliano D, Maiorino MI, Longo M, Bellastella G, Chiodini P, Esposito K. Type 2 diabetes and risk of heart failure: a systematic review and metaanalysis from cardiovascular outcome trials. Endocrine. 2019;65:15-24

4. Garg V, Verma S, Connelly K. Mechanistic insights regarding the role of SGLT2 inhibitors and GLP1 agonist drugs on cardiovascular disease in diabetes. Prog Cardiovasc Dis. 2019;62:349-57.

5. Giugliano D, Bellastella G, Longo M, et al. Relationship between improvement of glycaemic control and reduction of major cardiovascular events in 15 cardiovascular outcome trials: a meta-analysis with meta-regression. Diabetes Obes Metab. 2020;22:1397-405.

6. Giugliano D, Longo M, Scappaticcio L, Caruso P, Esposito K. Sodium-glucose transporter-2 inhibitors for prevention and treatment of cardiorenal complications of type 2 diabetes. Cardiovasc Diabetol. 2021;20:17.

7. Inzucchi SE, Zinman B, Fitchett $D$, et al. How does empagliflozin reduce cardiovascular mortality? Insights from a mediation analysis of the EMPAREG OUTCOME trial. Diabetes Care. 2018:41:356-63.

8. McMurray JJV, Solomon SD, Inzucchi SE, et al. Dapagliflozin in patients with heart failure and reduced ejection fraction. N Engl J Med. 2019;381:1995-2008.

9. Liberati A, Altman DG, Tetzlaff J, et al. The PRISMA statement for reporting systematic reviews and meta-analyses of studies that evaluate health care interventions: explanation and elaboration. Ann Intern Med. 2009;151:W65-94.

10. Higgins JP, Altman DG, Gøtzsche PC, et al. The Cochrane Collaboration's tool for assessing risk of bias in randomised trials. Br Med J. 2011;343:d5928.

11. Borenstein M, Higgins JP, Hedges LV, Rothstein HR. Basics of meta-analysis: I 2 is not an absolute measure of heterogeneity. Res Synth Methods. 2017:8:5-18.

12. Langan D, Higgins JPT, Simmonds M. Comparative performance of heterogeneity variance estimators in meta-analysis: a review of simulation studies. Res Synth Methods. 2017:8:181-98.

13. Egger M, Smith GD, Schneider M, Minder C. Bias in meta-analysis detected by a simple, graphical test. Br Med J. 1997;315:629-34.

14. Duval SJ, Tweedie RL. A non-parametric "trim and fill" method of accounting for publication bias in meta-analysis. J Am Stat Assoc. 2000;95:89-98.

15. Zinman B, Wanner C, Lachin JM, et al. Empagliflozin, cardiovascular outcomes, and mortality in type 2 diabetes. N Engl J Med. 2015;373:2117-28.

16. Neal B, Perkovic V, Mahaffey KW, et al. Canagliflozin and cardiovascular and renal events in type 2 diabetes. N Engl J Med. 2017:377:644-57.

17. Wiviott SD, Raz I, Bonaca MP, et al. Dapagliflozin and cardiovascular outcomes in type 2 diabetes. N Engl J Med. 2019;380:347-57.

18. Perkovic V, Jardine MJ, Neal B, et al. Canagliflozin and renal outcomes in type 2 diabetes and nephropathy. N Engl J Med. 2019;380:2295-306.

19. Heerspink HJL, Stefánsson BV, Correa-Rotter R, et al. Dapagliflozin in patients with chronic kidney disease. N Engl J Med. 2020;383:1436-46.

20. Cannon CP, Pratley R, Dagogo-Jack S, et al. Cardiovascular outcomes with ertugliflozin in type 2 diabetes. N Engl J Med. 2020;383(15):1425-35.

21. Packer M, Anker SD, Butler J, et al. Cardiovascular and renal outcomes with empagliflozin in heart failure. N Engl J Med. 2020;383:1413-24.

22. Bhatt DL, Szarek M, Pitt B, Cannon CP, et al. Sotagliflozin in patients with diabetes and chronic kidney disease. N Engl J Med. 2021;384(2):129-39.

23. Bhatt DL, Szarek M, Steg PG, et al. Sotagliflozin in patients with diabetes and recent worsening heart failure. N Engl J Med. 2021;384:117-28.
24. Anker SD, Butler J, Filippatos G, et al. Empagliflozin in heart failure with a preserved ejection fraction. N Engl J Med. 2021;385:1451-61.

25. Wanner C, Inzucchi SE, Lachin JM, et al. Empagliflozin and progression of kidney disease in type 2 diabetes. N Engl J Med. 2016;375:323-34.

26. Mahaffey KW, Jardine MJ, Bompoint S, et al. Canagliflozin and cardiovascular and renal outcomes in type 2 diabetes mellitus and chronic kidney disease in primary and secondary cardiovascular prevention groups. Circulation. 2019;140:739-50.

27. Petrie MC, Verma S, Docherty KF, et al. Effect of dapagliflozin on worsening heart failure and cardiovascular death in patients with heart failure with and without diabetes. JAMA. 2020;323:1353-68.

28. Jhund PS, Solomon SD, Docherty KF, et al. Efficacy of dapagliflozin on renal function and outcomes in patients with heart failure with reduced ejection fraction. Results of DAPA-HF. Circulation. 2021;143:298-309.

29. Wheeler DC, Stefánsson BV, Jongs N, et al. Effects of dapagliflozin on major adverse kidney and cardiovascular events in patients with diabetic and non-diabetic chronic kidney disease: a prespecified analysis from the DAPA-CKD trial. Lancet Diabetes Endocrinol. 2021;9:22-31.

30. McGuire DK, Shih WJ, Cosentino F, et al. Association of SGLT2 inhibitors with cardiovascular and kidney outcomes in patients with type 2 diabetes: A meta-analysis. JAMA Cardiol. 2021;6:148-58.

31. Salah HM, Al'Aref SJ, Khan MS, et al. Effect of sodium-glucose costrasporter 2 inhibitors on cardiovascular and kidney outcomes-Systematic review and meta-analysis of randomized placebo-controlled trials. Am Heart J. 2021;232:10-22

32. Giugliano D, Longo M, Caruso P, Maiorino MI, Bellastella G, Esposito K. Sodium-glucose co-transporter inhibitors for the prevention of cardiorenal outcomes in type 2 diabetes: an updated meta-analysis. Diabetes Obes Metab. 2021;23:1672-6.

33. Packer M, Butler J, Zannad F, et al. Empagliflozin and major renal outcomes in heart failure. N Engl J Med. 2021;385:1531-3.

34. Drazner MH. SGLT2 inhibition in heart failure and preserve ejection fraction-a win against a formidable foe. N Engl J Med. 2021;385:1522-4.

35. Zannad F, Ferreira JP, Pocock SJ, et al. SGLT2 inhibitors in patients with heart failure with reduced ejection fraction: a meta-analysis of the EMPEROR-Reduced and DAPA-HF trials. Lancet. 2020;396:819-29.

36. Qiu M, Ding L-L, Zhou H-R. Factors affecting the efficacy of SGLT2is on heart failure events: a meta-analysis based on cardiovascular outcome trials. Cardiovasc Diagn Ther. 2021;11:699-706.

37. GriffinM RaoVS, Ivey-Miranda J, et al. Empagliflozin in heart failure: diuretic and cardio-renal effects. Circulation. 2020;142:1028-39.

38. Ferrannini E, Mark M, Mayoux E. CV protection in the EMPA-REG OUTCOME Trial: a "Thrifty Substrate" hypothesis. Diabetes Care. 2016;39:1108-14.

39. Motoaki S, Shinichi G. Possible mechanism of hematocrit elevation by sodium glucose cotransporter 2 inhibitors and associated beneficial renal and cardiovascular effects. Circulation. 2019;139:1985-7.

40. Custodio JS, Duraes AR, Abreu M, et al. SGLT2 inhibition andheart failure-current concepts. Heart Fail Rev. 2018;23:409-18.

41. Nespoux J, Vallon V. SGLT2 inhibition and kidney protection. Clin Sci. 2018;132:1329-39.

42. http://www.fda.gov/news-events/press-announcements/fda-approvesnew-treatment-type-heart-failure.

43. http://www.fda.gov/news-events/press-announcements/fda-approvesjardiance-reduce-cardiovascular-death-adults-type-2-diabetes.

\section{Publisher's Note}

Springer Nature remains neutral with regard to jurisdictional claims in published maps and institutional affiliations. 